\title{
Stapled Bowel Anastomoses in Newborn Surgery
}

\author{
Yury Kozlov ${ }^{1,2}$ Vladimir Novogilov $^{2} \quad$ Alexey Podkamenev $^{1} \quad$ Irina Weber $^{1}$ \\ ${ }^{1}$ Department of Newborn Surgery, Pediatric Hospital, Irkutsk, Russian \\ Federation \\ 2 Department of Pediatric Surgery, Irkutsk State Academy of Medical \\ Continuing Education, Irkutsk, Russian Federation \\ Address for correspondence and reprint requests Yury Kozlov, MD, \\ Department of Newborn Surgery, Pediatric Hospital, 57 Sovetskaya \\ Street, Irkutsk 664009, Russian Federation \\ (e-mail: yuriherz@hotmail.com).
}

Eur J Pediatr Surg 2013;23:63-66.

\begin{abstract}
Keywords

- stapled intestinal anastomosis

- newborn

- children

Background In this work, we have described our experience with repair of bowel discontinuity by applying stapled anastomosis. The aim of this study was to compare two basic intestinal anastomotic techniques and to establish the feasibility of the stapled connection of bowel in neonates and infants.

Methods Between August 2009 and December 2011, we have performed 23 cases of hand-sewn anastomosis (group I) and 21 cases of mechanical anastomosis (group II) in children during the first 3 months of life. The standard hand technique was typically fashioned by continuous two-layer absorbable suture. A stapled anastomosis was accomplished using the linear endoscopic stapler with 2.5-mm staples (Ethicon EndoSurgery, Cincinnati, Ohio, United States). The two groups were compared for patients' demographics, operative report, and postoperative outcomes.

Results There were no differences in the preoperative parameters between the two groups regarding age before surgery, and body weight at operation. The "hand" group and "stapler" group had similar mean preoperative age (19.7 vs. 23.2 days; $p=0.096$ ) and average weight (2884.3 vs. $2716.0 \mathrm{~g} ; \mathrm{p}=0.692$ ). The mean operative time for bowel continuity restoration in group I was 77.4 minutes. In contrast, the mean duration of the operation in the group II was 56.4 minutes. The differences between groups were statistically significant $(p<0.0001)$. The mean time of full enteral feeding for patients with mechanical anastomosis was isochronous for patients with standard hand-sewn technique (6.7 vs. 6.7 days; $p=0.926$ ). The statistically identical postoperative hospital stay was registered in patients of both groups ( 13.3 vs. 14.1 days; $p=0.852$ ). The postoperative course was uneventful in all the patients. All the anastomoses were fully functional at the end of the study.

Conclusion Efficacy of the stapled anastomosis was confirmed by the shorter time of operation with saving of equal time of full enteral feeding and hospitalization.
\end{abstract}

\section{Introduction}

The creation of a join between two bowel ends in newborns and infants is a central surgical procedure in the life of the pediatric surgeons and has been usually performed using hand-sewn techniques. Since the introduction of stapling in infants younger than 4 months $^{1}$ only few articles reported usage of stapled technique in small patients. In this work, we have described our experience with repair of bowel discontinuity by applying stapled anastomosis. The aim of this study was to compare two basic intestinal anastomotic techniques and to establish the feasibility of the stapled connection of bowel in neonates and infants. received

May 15, 2012

accepted after revision

July 18, 2012

published online

October 25, 2012 


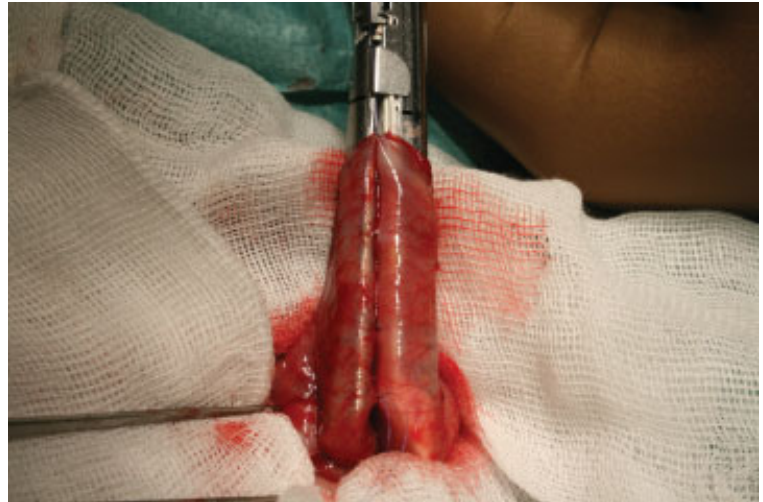

Figure 1 Creation of a first longitudinal line of stapled intestinal anastomosis (single stapled).

\section{Methods}

A retrospective review of the records of 44 infants younger than 3 months of age who underwent intestinal anastomoses from August 2009 to December 2011 was performed. The ethical committee of the Irkutsk Pediatric Hospital (Irkutsk, Russia) approved this study.

The standard perioperative data and outcome variables included age and weight at operation, operative time and time of full enteral nutrition, and length of hospital stays. Differences between groups were assessed using the MannWhitney U Test. A $p$ value of less than 0.05 was considered statistically significant.

In all cases of bowels join (group II), a stapled side-to-side functioning end-to-end intestinal anastomosis was constructed using the Endopath ATW-35 Endoscopic Articulating Linear Stapler with 2.5-mm staples (Ethicon Endo-Surgery, Cincinnati, Ohio, United States). Standard double-stapled surgical techniques described in pediatric surgical literature were used for creation of the intestinal anastomoses. ${ }^{2,3}$

In brief, after efferent bowel limbs were sutured in proximity, the stapled device cannulated the bowel limbs. Before closing the stapler and completing the anastomosis, the stapler was positioned onto the antimesenteric wall of the intestine. After being inspected for intestinal lumens and patency, the stapler was again applied across the joined bowel limbs, closing the bowel anastomosis (-Figs. 1 and 2). Staple

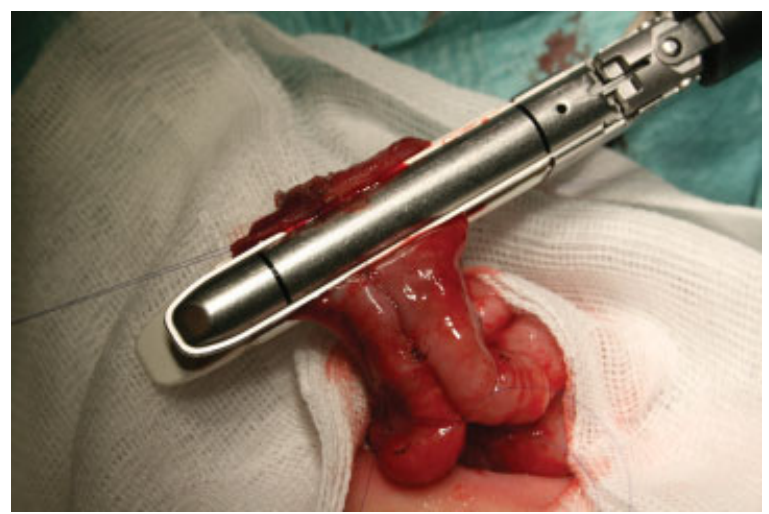

Figure 2 Complete formation of a stapled side-to-side functioning end-to-end intestinal anastomosis (double stapled). lines were not oversewn unless there was evidence of a leak at the time of operation.

The minimum body weight of the patient in the "stapler" group was $1210 \mathrm{~g}$. In the current study, use of the suturing device was defined not by the weight of the patient, but by the diameter of connected segments of intestines. The endoscopic stapler was used for this manipulation because only this device can be inserted in a small-caliber gut lumen of the neonate and premature children. The proximal intestinal limb having the larger size was cannulated with larger end of the stapler. The smaller end of the suturing device was inserted in other distal intestinal segment. Possibility of application of the stapler was defined during operation by measurement of diameter of the smallest segment of the gut. We calculated perimeter of cross-section of the small end of the stapler and defined that the smallest diameter of a gut for anastomosis will be $6 \mathrm{~mm}$. Only in these cases we opened sterile box of the device and applied the mechanical suture.

In the "hand-sewn" patients (group I), anastomoses were performed in an end-to-end fashion by continuous two-layer absorbable suture material.

We have no selection criteria between groups. Choice of anastomotic technique was determined by preference of the senior surgeon.

The length of the follow-up averaged 6 months. The patients were returned 1 month to 1 year after surgery for repeated medical examination. The bowel function, body weight, and gastrointestinal symptoms were recorded at surgical service of the pediatric hospital.

\section{Results}

A total of 44 patients were identified during the study period. A total of 23 patients of group I had two-layer hand-sewn anastomosis and 21 patients of the group II had stapled sideto-side functional end-to-end intestinal anastomosis. The reasons for the anastomoses in hand-sewn group included necrotizing enterocolitis (NEC) and isolated ileal perforations (IIP) (60.9\%), patent omphalomesenteric duct (17.4\%), and congenital and acquired bowel obstruction (21.7\%). The most common diagnoses for patients undergoing stapled anastomoses were NEC and IIP (52.4\%), anal atresia (14.3\%), bowel necrosis in gastroschisis (9.5\%), patent omphalomesenteric duct (9.5\%), intestinal duplications (9.5\%), and total Hirschsprung disease (4.8\%). The patient's data and outcome are summarized in - Table 1.

There were no differences in the preoperative data between the two groups regarding the age before surgery and body weight at surgery. The "hand" and "stapler" groups had similar mean preoperative age (19.7 vs. 23.2 days; $p=0.096$ ) and average weight ( 2884.3 vs. $2716.0 \mathrm{~g} ; p=0.692$ ). The mean time to full enteral feeding for patients with stapled anastomosis was isochronous for patients with standard hand-sewn technique (6.7 vs. 6.7 days; $p=0.926$ ). The statistical identical postoperative hospital stay was registered in patients of both groups ( 13.3 vs. 14.1 days; $p=0.852$ ).

The only statistically significant parameter was the operative time. The mean operative time for the restoration of 
Table 1 A comparison of preoperative and postoperative details in the groups

\begin{tabular}{|l|l|l|l|}
\hline Parameters & Group I: hand-sewn anastomosis & Group II: stapled anastomosis & $\begin{array}{l}p \text { Values } \\
\text { Mann-Whitney U test }\end{array}$ \\
\hline Preoperative parameters & $19.7\left(2.8^{\mathrm{a}}\right)$ & $23.2(2.8)$ & 0.095516 \\
\hline Age at operation (day) & $2716.0(226.0)$ & 0.692459 \\
\hline Body weight at operation (g) & $2884.3(240.3)$ & $56.4(2.1)$ & $<0.0001$ \\
\hline Postoperative parameters & $77.4(2.4)$ & $6.7(0.6)$ & 0.92589 \\
\hline Operative time (min) & $6.7(0.5)$ & $14.1(1.5)$ & 0.852406 \\
\hline Full enteral feeding (day) & $13.3(1.0)$ & \\
\hline Length of hospital stay (day) &
\end{tabular}

aalues are expressed as mean (SEM).

bowel continuity was 77.4 minutes in group I and 56.4 minutes in group II $(p<0.0001)$.

The postoperative course was routine in all patients of both groups. There were no intra- and postoperative complications. We had no anastomotic leak and wound infections.

At follow-up, bowel function was perfect in all operated patients. The infants were free from late postoperative complications such as stricture and failure in area of anastomosis. Good intestinal transit and absorption were observed at these patients.

\section{Discussion}

Intestinal anastomosis is a surgical procedure to establish communication between two portions of the intestine. In neonatal and pediatric patients, it may be required for the management of many conditions. Some conditions may require resection of pathology followed by primary anastomosis, whereas other conditions may necessitate delayed anastomosis.

Intestinal anastomosis can be performed by a hand-sewn technique using absorbable or nonabsorbable sutures or stapling devices. Although surgical stapling devices have existed since the early 20th century, their use in routine gastrointestinal surgery has not been widespread until $\sim 30$ years ago when their design became much more efficient and convenient.

Among the American and European surgeons who were trained in surgery at the end of the 20th century, staplers were called as "Russian Gun." The history of the origin of this term is known. ${ }^{4}$ The Russian Scientific Institute for Surgical Devices and Instruments studied and then developed stapling instruments that served as the prototype of today's staplers. An American Surgeon, Dr. Mark Ravitch, visited Soviet Union and observed Russian surgeons who operated with a stapler. As a result, Dr. Ravitch studied this surgical product and then designed a series of American instruments with reusable staplers and sterilized cartridges. That is how the era of stapled anastomoses in surgery began. With modern devices, the staple lines are of more consistent quality and anastomoses in difficult locations are easier to construct.
The role of stapling devices in surgery has continued to expand. In adults, the safety and efficacy of stapled intestinal anastomosis have been extensively documented since $1978 .^{5}$ Recent studies indicate that patients requiring mechanical anastomoses who are managed with stapling have a similar rate of complications than do those in whom a hand-sewn anastomosis is used. A Cochrane review of seven trials found fewer leaks after stapled anastomosis with no differences in operative time or the incidence of stricture or wound infection. ${ }^{6}$ In addition, a meta-analysis studied 13 trials reported no differences in mortality and leakage rate after stapling usage. 7

In pediatric surgery, gastrointestinal staplers were applied traditionally and double-stapled anastomotic technique was performed by anatomic side-by-side functioning and end-toend anastomosis was used. Several studies have described the use of staplers in children for the treatment of Hirschsprung disease, ${ }^{8}$ appendectomy, ${ }^{9}$ Meckel diverticulectomy, ${ }^{10}$ and intestinal lengthening procedures. ${ }^{11}$

There are four articles describing the use of intestinal stapler in infants for bowels join. ${ }^{1-3,12}$ In 1995, Powell ${ }^{1}$ published a series of seven newborns with a mean age of 72 days and a mean weight of $3.7 \mathrm{~kg}$. In our study, mean age at operation in "stapler" group was 23 days and mean weight was $2.7 \mathrm{~kg}$. Patients in "stapler" group were also younger compared with report of Wrighton et $\mathrm{al}^{2}$ (mean age 105 days).

Although the superiority of stapled anastomosis in newborns is not proven yet over hand-sewn anastomosis, it has definitely facilitated the ease of doing the procedure. In 2011, Mitchell et $\mathrm{al}^{3}$ reported a series of using staplers in 64 small children with phenomenal postoperative results excluding leaks. Mitchell et al concluded that the stapled anastomosis is an effective alternative for restoration of bowel discontinuity in newborns and small babies when permitted by intestinal size.

Another benefit of stapler anastomosis, which has been shown in our results and support previous reports, is clear decrease in operative time. This may be particularly important in small, premature infants.

In modern literature, complications of mechanical method of join of bowel are described rarely. There is only one report 
in the published articles of adverse outcomes after stapled intestinal anastomoses in children presented with intestinal volvulus several years after stapled anastomosis at 2 months and 3 years of age. ${ }^{13}$ We have not found any complications in our groups in short-term follow-up.

In conclusion, we reported series of stapled intestinal anastomoses in infants and compared outcomes with those who underwent hand-sewn bowel connection. Efficacy of the stapled anastomosis was confirmed by the shorter operative time with saving equal time to full enteral feeding and length of hospital stay. We did not observe complications in both groups at operations and short-term follow-up. We concluded that the age and size of patients are not the limiting factors for using gastrointestinal staplers. Our study further validates the use of the stapled anastomosis in neonates, but investigation is required to verify long-term follow-up.

\section{Acknowledgments}

This article is addressed to former successes of Russian researchers in the field of the stapling devices; the modern design was developed in the former Soviet Union. ${ }^{14}$

\section{Conflict of Interest}

None

\section{References}

1 Powell RW. Stapled intestinal anastomosis in neonates and infants: use of the endoscopic intestinal stapler. J Pediatr Surg 1995;30(2):195-197

2 Wrighton L, Curtis JL, Gollin G. Stapled intestinal anastomoses in infants. J Pediatr Surg 2008;43(12):2231-2234
3 Mitchell IC, Barber R, Fischer AC, Schindel DT. Experience performing 64 consecutive stapled intestinal anastomoses in small children and infants. J Pediatr Surg 2011;46(1):128-130

4 Baker RS, Foote J, Kemmeter P, Brady R, Vroegop T, Serveld M. The science of stapling and leaks. Obes Surg 2004;14(10): 1290-1298

5 Chassin JL, Rifkind KM, Sussman B, et al. The stapled gastrointestinal tract anastomosis: incidence of postoperative complications compared with the sutured anastomosis. Ann Surg 1978;188 (5):689-696

6 Choy PY, Bissett IP, Docherty JG, et al. Stapled versus handsewn methods for ileocolic anastomosis. Cochrane Database Syst Rev 2011;(9):CD004320

7 MacRae HM, McLeod RS. Handsewn versus stapled anastomoses in colon and rectal surgery: a meta-analysis. Dis Colon Rectum 1998;41(2):180-189

8 Talbert JL, Seashore JH, Ravitch MM. Evaluation of a modified Duhamel operation for correction of Hirschsprung's disease. Ann Surg 1974;179(5):671-675

9 Olguner M, Akgür FM, Uçan B, Aktuğ T. Laparoscopic appendectomy in children performed using single endoscopic GIA stapler for both mesoappendix and base of appendix. J Pediatr Surg 1998;33 (9):1347-1349

10 Valla JS, Steyaert H, Leculée R, Pebeyre B, Jordana F. Meckel's diverticulum and laparoscopy of children. What's new? Eur J Pediatr Surg 1998;8(1):26-28

11 Javid PJ, Kim HB, Duggan CP, Jaksic T. Serial transverse enteroplasty is associated with successful short-term outcomes in infants with short bowel syndrome. J Pediatr Surg 2005;40(6):1019-1023, discussion 1023-1024

12 Simmons JD, Gunter JW III, Manley JD, Sawaya DE, Blewett CJ. Stapled intestinal anastomosis in neonates: validation of safety and efficacy. Am Surg 2010;76(6):644-646

13 Jackson CC, Bettolli MM, De Carli CF, Bass J, Rubin SZ, Sweeney B. Beware of stapled side-to-side bowel anastomoses in small children. Eur J Pediatr Surg 2007;17(6):426-427

14 Astafiev GV. Investigation process relating to tissue compression in suturing and stapling apparatus. Surgical Staplers (Chirurgiceskiye Shivayushiye Apparaty) 1967:22-31 